\title{
Tumor Treating Fields in the Management of Patients with Malignant Gliomas
}

\author{
Ashley P. Ghiaseddin, MD* \\ David Shin, MD \\ Kaitlyn Melnick, MD \\ David D. Tran, MD, $P h D^{*}$
}

\author{
Address \\ *Lillian S. Wells Department of Neurosurgery, University of Florida College of \\ Medicine, 1505 SW Archer Road, Gainesville, FL, 32608, USA \\ Email: ashley.ghiaseddin@neurosurgery.ufl.edu \\ Email: david.tran@neurosurgery.ufl.edu
}

Published online: 30 July 2020

(C) Springer Science+Business Media, LLC, part of Springer Nature 2020

This article is part of the Topical Collection on Neuro-oncology

Keywords Tumor treating fields · CNS tumors · Malignant glioma · Glioblastoma $\cdot$ Alternating electric fields · Optune ${ }^{\circledR}$

\section{Opinion statement}

Malignant gliomas remain a challenging cancer to treat due to limitations in both therapeutic and efficacious options. Tumor treating fields (TTFields) have emerged as a novel, locoregional, antineoplastic treatment modality with favorable efficacy and safety being demonstrated in the most aggressive type of malignant gliomas, glioblastoma (GBM). In 2 large randomized, controlled phase 3 trials, the addition of TTFields was associated with increased overall survival when combined with adjuvant temozolomide (TMZ) chemotherapy in patients with newly diagnosed GBM (ndGBM) and comparable overall survival compared with standard chemotherapy in patients with recurrent GBM (rGBM). TTFields target cancer cells by several mechanisms of action (MoA) including suppression of proliferation, migration and invasion, disruption of DNA repair and angiogenesis, antimitotic effects, and induction of apoptosis and immunogenic cell death. Having several MoAs makes TTFields an attractive modality to combine with standard, salvage, and novel treatment regimens (e.g., radiotherapy, chemotherapy, and immunotherapy). Treatment within the field of malignant gliomas is evolving to emphasize combinatorial approaches that work synergistically to improve patient outcomes. Here, we review the current use of TTFields in GBM, discuss MOA and treatment delivery, and consider the potential for its wider adoption in other gliomas. 
Primary central nervous system (CNS) cancers account for $1.6 \%$ of cancers diagnosed each year worldwide, with gliomas being the most common histological type $[1,2]$. The incidence of gliomas, primary brain tumors originating from glial or neuronal precursor cells, increases with age, with highest rates in those 75 years and older [3]. High-grade, malignant gliomas represent $35-45 \%$ of primary brain tumors and include glioblastoma (GBM), anaplastic astrocytoma, and anaplastic oligodendroglioma [4]. GBM, constituting $60-70 \%$ of malignant gliomas, is a highly aggressive, WHO grade IV glioma with a dismal 5-year survival rate of $6.8 \%$ [4-6]. Due to its aggressive and diffusely infiltrative nature, recurrence is common, often leading to rapid tumor spread to other brain regions [6], while the blood-brain barrier (BBB) typically limits metastatic spread beyond the brain [7]. GBM is highly debilitating as symptoms such as nausea, weakness, seizures, and headaches can be severe. Neurocognitive and personality changes, aphasia, vision problems, loss of gait, thromboembolic events, and hemiparesis may occur depending on tumor location and the extent of tumor spread and associated edema [8].

Despite the expansion in therapeutic options for malignant gliomas in the decade following the approval of TMZ, long-term survival rates remain dismal. Further stoking a keen demand for new therapeutic approaches to reverse this bleak trend is the increasing prevalence of primary CNS tumors in the general population (a $17.3 \%$ increase globally between 1990 and 2016), likely due to an aging population [2]. From this discouraging background emerged tumor treating fields (TTFields), a novel locoregional antineoplastic treatment modality utilizing low-intensity (1$3 \mathrm{~V} / \mathrm{cm})$, intermediate frequency $(\sim 100-500 \mathrm{kHz})$, alternating electric fields with unique MOA. TTFields are delivered through 2 pairs of orthogonal transducer arrays $[9,10]$. Array positioning is individualized to maximize therapeutic delivery of fields at the tumor bed [11], affording a targeted approach with negligible adverse effects on normal CNS functions [12].
Prior to TTFields, the standard treatment for ndGBM (i.e., the Stupp protocol) included maximal safe debulking surgery followed by radiotherapy (RT) plus concomitant TMZ and adjuvant maintenance TMZ [13, 14]. The Stupp protocol improved median OS to 14.6 months vs 12 months with RT alone [13]. Treatment options for rGBM were less well defined, encompassing additional surgery, cytotoxic chemotherapy (e.g., other alkylating agents such as carmustine and lomustine and platinumbased regimens), repeat irradiation, bevacizumab (a vascular endothelial growth factor A [VEGFA] inhibitor), investigational therapies, and palliative/best supportive care (for patients with poor performance status) $[14,15]$. Neither repeat surgery $[16,17]$ nor bevacizumab $[18,19]$ has been demonstrated to prolong survival, while cytotoxic chemotherapy including TMZ re-challenge showed variable efficacy [20-22] in phase 3 trials. Moreover, these approaches are associated with systemic adverse events (AEs) that negatively impact quality of life (QoL) and have limited use for patients with poor functional status and/or multiple comorbidities [23-25].

In the phase 3 EF-14 trial of ndGBM, TTFields plus maintenance TMZ significantly improved median overall survival (OS) relative to TMZ alone (20.9 vs 16.0 months, respectively; $p<0.001$ ) [26]. In the phase 3 EF-11 trial of rGBM, TTFields monotherapy vs physicians' choice chemotherapy showed comparable survival benefits (6.6 vs 6.0 months, respectively), although the study was

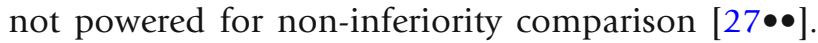
Both trials reported favorable safety profiles [26, 27]. Based on these results, TTFields $(200 \mathrm{kHz})$ are approved for adult patients with rGBM as monotherapy and for ndGBM in combination with adjuvant TMZ in the USA, Europe, Japan, Israel, and Hong Kong, China [11, 28]. The National Comprehensive Cancer Network ${ }^{\circledR}$ guidelines recommend TTFields for ndGBM with category 1 evidence and rGBM with category 2B evidence [15] and the American Society of Clinical Oncology recognizes TTFields as a novel treatment device 
for brain cancer [29]. Herein, we review the use of TTFields for GBM, discuss mechanisms of action
(MoA) and treatment delivery, and consider the potential for its wider use.

\section{Mechanisms of action of TTFields (Fig. 1)}

\section{Antimitotic effects of TTFields}

The selective antimitotic effects on proliferating cells with minimal impact on nonproliferating cells establish the foundation for the anticancer properties of TTFields [9]. TTFields perturb cells in mitosis resulting in plasma membrane contractions and instability and the formation of plasma membrane blebbing [9]. During metaphase of the cell cycle, TTFields affect motility and assembly of intracellular macromolecules required for mitotic spindle formation, leading to mitotic catastrophe, chromosomal breakage forming micronuclei, and cell death [9]. During anaphase, telophase, and cytokinesis, TTFields disrupt polarity and alignment of several cellular structures (e.g., the spindle structure and contractile ring), preventing complete cytoplasmic separation, which results in either apoptosis via p53-dependent and p53-independent processes or generation of aberrant daughter cells [32-34]. The ability of TTFields to target multiple phases of the cell cycle may create additive or synergistic effects with other therapies that disrupt Gap 1/synthesis (G1/S) or Gap $2 /$ mitosis (G2/M) phases of the cell cycle, allowing for broader targeting of cancer cell mitosis.

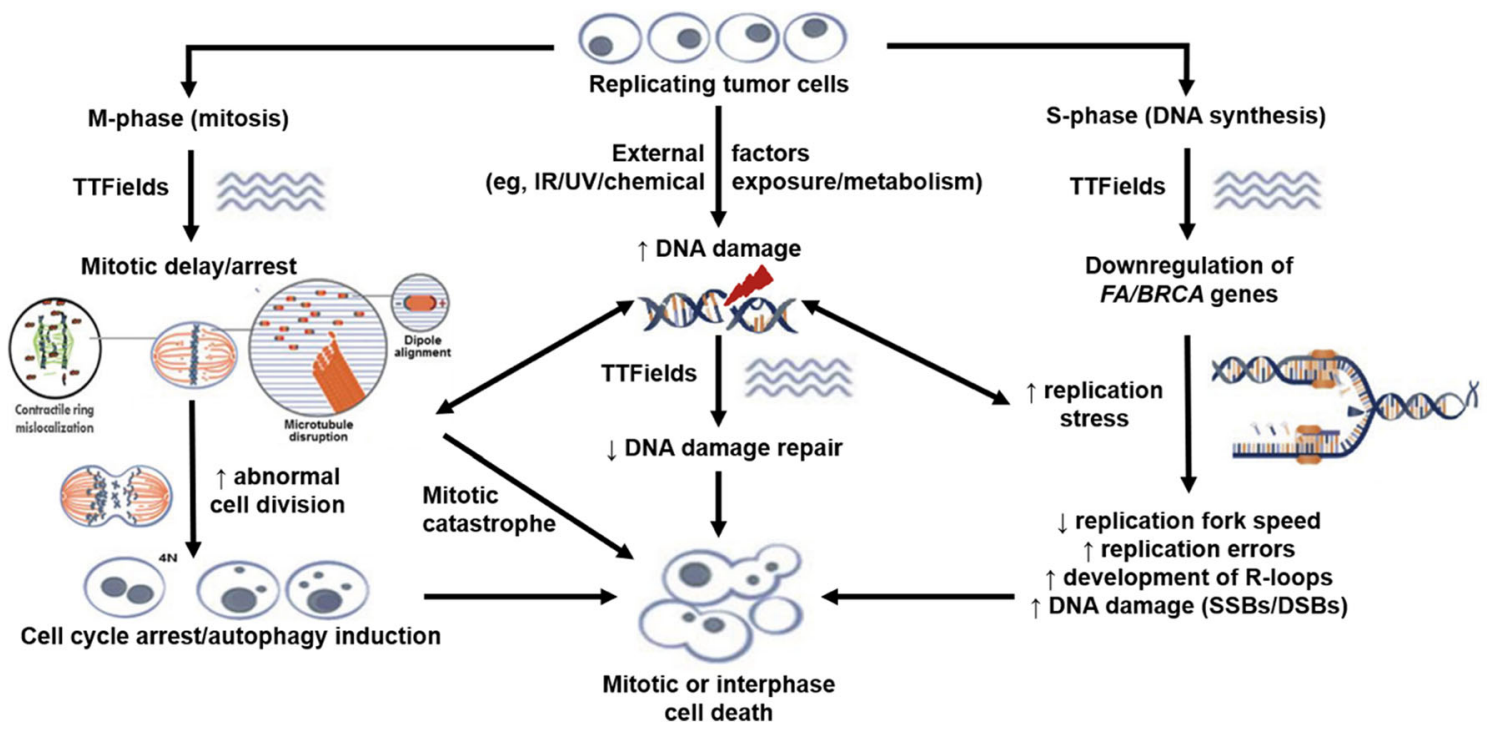

Fig. 1. TTFields mechanism of action. DSB, double-strand break; IR, infrared; SSB, single-strand break; TTFields, tumor treating fields; UV, ultraviolet. Novocure 2018; Image was adapted with permission [30, 31]. 


\section{TTFields-triggered programmed cell death}

Many mechanisms have been put forth to explain how TTFields induce programmed cell death. In one scenario, TTFields interfere with DNA fork replication and induce movement and repositioning of DNA fragments created during replication, leading to widespread disruption of the DNA damage repair and breast cancer 1 (BRCA1)-mediated homologous recombination pathways [35-37]. In another, endoplasmic reticulum stress in mitotic cells under the effects of TTFields may trigger adenosine monophosphate-activated protein kinase-dependent autophagy [38] and/or immunogenic cell death [39-41]. The latter may also be a direct result of cytoplasmic double-stranded DNA released from TTFields-generated micronuclei, leading to activation of innate immunity such as the STING pathway and pyroptosis, a highly inflammatory form of programmed cell death, and release of pro-inflammatory cytokines and chemokines as well as type 1 interferon response pathways [39, 42].

\section{Effects of TTFields on cell migration and membrane integrity}

TTFields have been shown to inhibit cell migration and invasion by inducing a more adhesive cell phenotype. This is achieved through dysregulation of cytoskeletal structures and proteins related to the epithelial-mesenchymal transition (e.g., actins, vimentin, and cadherin), potentially reducing the likelihood of recurrence or metastases [43-46]. TTFields also increase permeability of the plasma membrane in human GBM cells in vitro by triggering mislocalization of the tight junction proteins Claudin- 5 and $\mathrm{ZO}-1$ from the plasma membrane to the cytoplasm $[47,48]$. Election microscopic images of the human GBM cell line U87-MG and murine astrocytoma cell line KR158B treated with TTFields for $1 \mathrm{~h}$ revealed a large number of perforations scattered throughout the plasma membrane $[47,48]$. These perforations were large enough to allow for the uptake of fluorescently labeled dextran particles of up to $20 \mathrm{kDa}$ in size. In rodent brains, TTFields induce temporary BBB disruption, lasting up to $96 \mathrm{~h}$ $[47,48]$. These findings provide a potential new application for TTFields to enhance CNS delivery of small molecule BBB-impermeant pharmacological agents. Additionally, increased cell membrane permeability in GBM cells was shown to facilitate better uptake of intraoperative agents such as 5aminolevulinic (5-ALA) used intraoperatively to delineate tumor-normal brain margins $[48 \bullet \bullet]$. Lastly, TTFields impair angiogenesis, a key process for tumor growth and progression, via VEGF-induced and hypoxia-inducible factor 1 alpha-regulated pathways [45], thus identifying potential synergism with antiangiogenic agents (e.g., bevacizumab, cediranib, enzastaurin, and sunitinib) $[49,50]$.

\section{Delivery parameters of TTFields}

Optimal frequencies for maximizing cytotoxic effects vary by tumor type and may inversely correlate with cell size [10]. For a specific frequency, the electrical intensity determines the degree of antimitotic cell death; a minimal threshold of $1 \mathrm{~V} / \mathrm{cm}$ was observed across tumor types [51], with confirmed dose-correlated clinical significance in survival in ndGBM [52•]. The electrical intensity reaching the target depends on tumor position and the conductivity and impedance of the surrounding tissues [53]. Transducer array positioning is individualized for each patient to ensure maximum field intensity at the tumor bed, with arrays 
replaced at minimum twice per week and, for GBM, the scalp re-shaved to maintain optimal contact and functioning [12]. Since measuring TTFields frequency and intensity at the target site in patients is not feasible, computergenerated models/algorithms are used to simulate the expected trajectory and electrical field properties [54] to optimize array size and layouts [55]. Realistic computational phantoms have been generated to model TTFields delivery to tumors in the brain [34,56-58] and torso [59].

In GBM, TTFields $(200 \mathrm{kHz})$ are delivered to the tumor bed noninvasively and continuously using the Optune ${ }^{\circledR}$ system (Fig. 2a) through 2 pairs of orthogonal transducer arrays affixed to the patient's shaved scalp (Fig. 2b) at the anterior-posterior and bitemporal axes with the specific placement locations depending on tumor location [12]. The Novocure Patient Transducer Array Layout (NovoTAL ${ }^{\mathrm{TM}}$ ) system is an FDA-approved software program that configures optimal transducer array layouts based on the patient head and tumor size and location, as determined by the axial and coronal $\mathrm{T} 1$ postcontrast sequences of brain MRI (Fig. 3) [12, 60, 61]. NovoTAL ensures that field intensity remains highest at the tumor bed and allows array adjustment to retarget tumors as they respond or progress $[12,60,61]$.

Since the fields alternate rapidly, TTFields have negligible effect on quiescent, terminally differentiated cells, including normal adult brain cells [9]. As TTFields are delivered locoregionally, they also have no effect on rapidly proliferating cells in the rest of the body, nor do they stimulate nerves or muscles since TTFields' frequency is much faster than the frequencies of physiologic fields found in these tissues $[9,10]$. In addition, field intensities generated by TTFields within target tissues are low and do not generate any appreciable local heating [10]. Thus, TTFields delivered according to NovoTAL mapping have both high targeting selectivity and low potential for local and systemic toxicity.

\section{Clinical data for TTFields in GBM}

\section{Clinical trials in rGBM and ndGBM}

In the phase 3 EF-11 trial for rGBM, TTFields monotherapy demonstrated comparable survival benefits and favorable safety, tolerability, and QoL versus physicians' choice chemotherapy (Table 1); however, the study was not pow-

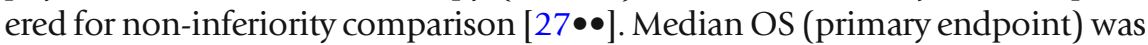
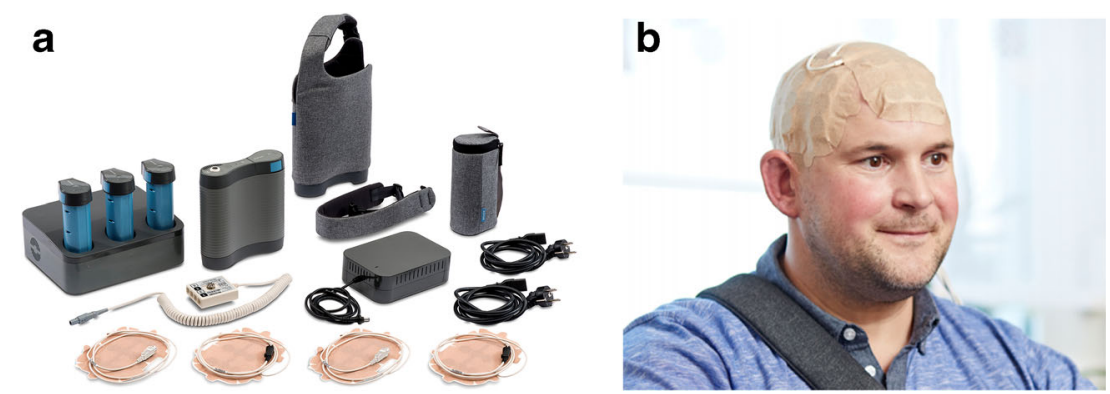

Fig. 2. The Optune ${ }^{\circledR}$ TTFields delivery system for GBM (a). Transducer arrays on a patient with GBM (b). GBM, glioblastoma; TTFields, tumor treating fields. Novocure 2019๑; all rights reserved. Permission for global image use was obtained from the patient. 


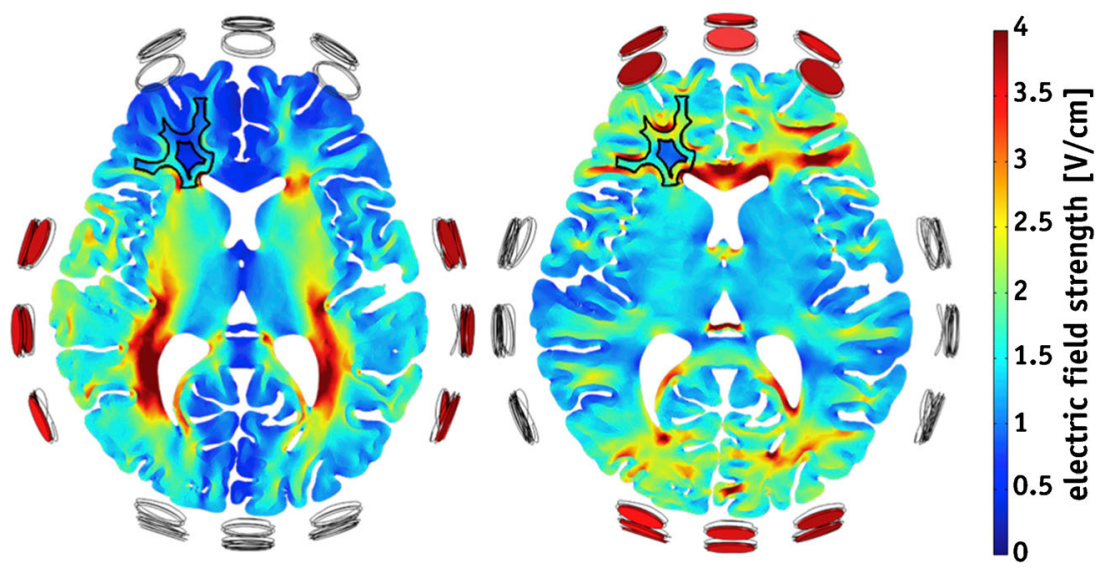

Fig. 3. Colormaps depicting the maximum TTFields intensity distributions in default layouts for brain tissue of a patient with pairs of transducer arrays positioned left-right (left) and anterior-posterior (right) in axial slices through the brain. TTFields, tumor treating fields. Image was reproduced with permission [55].

6.6 vs 6.0 months with TTFields versus chemotherapy, respectively (HR 0.86 [95\% CI 0.66-1.12]; $p=0.27$ ), and 6-month progression-free survival (PFS) was $21.4 \%$ versus $15.1 \%$, respectively $(p=0.13)[27 \bullet \bullet$. The most common AEs with TTFields were mild-to-moderate contact dermatitis beneath the arrays $(16 \%)[27 \bullet \bullet]$. In comparison, there were significantly more grade $\geq 2$ AEs, including gastrointestinal (17\%), hematological (17\%), and infectious (8\%) AEs reported with chemotherapy than with TTFields $(4 \%, 3 \%$, and $4 \%$, respec-

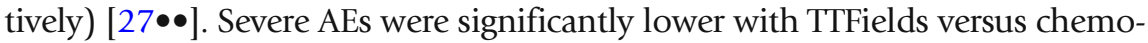
therapy ( $6 \%$ vs $16 \%$, respectively; $p=0.022$ ) [27••].

Table 1. Summary of efficacy outcomes for phase 3 TTFields Adult GBM clinical trials and patient registry database

\begin{tabular}{|c|c|c|c|c|c|}
\hline \multirow[t]{2}{*}{$\begin{array}{l}\text { Treatment } \\
\text { Arms }\end{array}$} & $\begin{array}{l}\mathrm{EF}-11(\mathrm{rGBM}) \\
N=237\end{array}$ & & $\begin{array}{l}\text { PRiDe (rGBM) } \\
N=457\end{array}$ & $\begin{array}{l}\text { EF-14 (ndGBM) } \\
N=695\end{array}$ & \\
\hline & $\begin{array}{l}\text { TTFields } \\
(n=120)\end{array}$ & $\begin{array}{l}\text { Chemotherapy } \\
(n=117)\end{array}$ & $\begin{array}{l}\text { TTFields } \\
(n=457)\end{array}$ & $\begin{array}{l}\text { TTFields + TMZ } \\
(n=466)\end{array}$ & $\begin{array}{l}\text { TMZ } \\
(n=229)\end{array}$ \\
\hline $\begin{array}{l}\text { Median OS, } \\
\text { months }\end{array}$ & 6.6 & 6.0 & 9.6 & 20.9 & 16.0 \\
\hline $\mathrm{HR}(95 \% \mathrm{CI}) ; p$ & $\begin{array}{l}0.86(0.66-1.12) \\
p=0.27\end{array}$ & & NA & $\begin{array}{c}0.63(0.53-0.76) \\
p<0.001\end{array}$ & \\
\hline $\begin{array}{l}\text { PFS at } 6 \\
\text { months, \% }\end{array}$ & 21.4 & 15.1 & NS & 56 & 37 \\
\hline $\begin{array}{l}\text { 2-year } \\
\text { survival, \% }\end{array}$ & 8 & 5 & 30 & 43 & 31 \\
\hline $\begin{array}{l}\text { 5-year } \\
\text { survival, \% }\end{array}$ & NS & NS & NS & 13 & 5 \\
\hline $\begin{array}{l}\text { Response } \\
\text { rate, \% }\end{array}$ & 14 & 9.6 & NS & NS & NS \\
\hline
\end{tabular}


In the EF-14 trial, significant TTFields survival benefits for patients with ndGBM were reported. Median PFS (primary endpoint) was significantly longer with TTFields plus maintenance TMZ than with TMZ alone (6.7 vs 4.0 months, respectively; HR 0.63 [95\% CI 0.53-0.76]; $p<0.001$ ), and 6-month PFS was $56 \%$ versus $37 \%$, respectively ( $p<0.001$; Table 1 ) [26]. Median OS (secondary endpoint) was 20.9 versus 16.0 months (HR 0.63 [95\% CI 0.53-0.76]; $p$ $<0.001$ ) with TTFields plus maintenance TMZ versus TMZ alone, respectively [26]. Two-year OS was $43 \%$ versus 31\% $(p<0.001)$, and 5 -year OS was $13 \%$ versus $5 \%(p=0.004)$ with TTFields plus TMZ versus TMZ alone, respectively [26]. AEs were reported in $48 \%$ of patients receiving combination therapy vs $44 \%$ with TMZ alone [26]. The most common TTFields-related AE was mild-tomoderate skin irritation beneath the arrays (52\% [severe in $2 \%$ ] vs $0 \%$ with TMZ alone), while chemotherapy was associated with systemic AEs [26].

Health-related QoL (HRQoL), a predefined endpoint in the EF-14 trial, did not differ significantly between treatment arms, except for itchy skin [62]. Deterioration-free survival was significantly longer (all $p<0.01$ ) with TTFields plus maintenance TMZ versus TMZ alone, respectively, for global health (4.8 vs 3.3 months), physical functioning (5.1 vs 3.7 months), emotional functioning (5.3 vs 3.9 months), pain (5.6 vs 3.6 months), and leg weakness (5.6 vs 3.9 months) [62]. Time to deterioration, reflecting treatment influence, differed significantly for itchy skin (shorter with TTFields; 8.2 vs 14.4 months; $p<0.001$ ) and pain (longer with TTFields; 13.4 vs 12.1 months; $p<0.01$ ). Role, social, and physical functioning were not affected by TTFields [62]. Estimated tolerability through activities of daily life and cognition using the Karnofsky performance score (KPS) and mini-mental state examination (MMSE) were evaluated. For KPS, time to a sustained 10-point decrease was significantly longer with TTFields plus maintenance TMZ (5.5 months vs 3.9 months, respectively; HR 0.80 [95\% CI 0.67-0.95]; $p=0.009)$ [26]. Time to a sustained 6-point decline in MMSE was also significantly longer with TTFields plus TMZ (16.7 months vs 14.2 months, respectively; HR 0.79 [95\% CI 0.66-0.95]; $p=0.01$ ) [26].

\section{Factors impacting clinical outcomes}

\section{Treatment adherence}

Since TTFields only exert a therapeutic effect when the device is actively utilized and, thus, do not have a half-life [63], increased treatment adherence is expected to be associated with improved survival in patients with GBM [64-66]. In a post hoc analysis of the EF- 11 trial, monthly maximal usage duration of $\geq 75 \%$ (i.e., $\geq 18 \mathrm{~h}$ /day averaged over a 4-week cycle) was associated with significantly longer median OS compared with a daily time of usage of $<75 \%$ (7.7 vs 4.5 months, respectively; $p=0.042$ ) [64]. A treatment adherence analysis of real-world rGBM registry data found a similar significant association between a $\geq 75 \%$ average daily usage rate and a higher median OS (13.5 vs 4.0 months, respectively; HR 0.43 [95\% CI 0.29-0.63]; $p<0.0001$ ] [63]. A subgroup analysis of the EF-14 trial found that a monthly threshold of 50\% TTFields usage time was needed with TTFields plus maintenance TMZ to show improved PFS (HR 0.70 [95\% CI 0.47-1.05]) and OS (HR 0.67 [95\% CI 0.45-0.99]) versus TMZ alone [66]. Similar to the EF-11 study, adherence to TTFields treatment with a monthly usage duration of $\geq 75 \%$ was associated with significantly longer median OS as compared with a monthly usage duration of $<75 \%$ (HR 
$0.78 ; p=0.031$ ), independent of sex, extent of resection, O-6-methylguanineDNA methyltransferase (MGMT) methylation status, age, region, and performance status [66]. Monthly usage time of $>90 \%$ had maximal survival benefit; for the TTFields plus TMZ arm compared with TMZ alone, median PFS was 8.2 months versus 4.0 months, respectively; median OS was 24.9 months versus 16.0 months, respectively (HR 0.52 [95\% CI $0.35-0.79 ; p=0.0007)$; and 5-year survival was $29.3 \%$ versus $4.5 \%$, respectively [66]. Of note, the second-generation Optune with several improvements (e.g., decreased weight, easy switch between plug-in and portable use for continuous treatment, and portable bags [67]) compared with the first-generation Optune, on which the EF-11 and EF-14 studies were based, enhances patient satisfaction and is associated with a higher treatment duration rate of patient adherence to TTFields [68].

\section{TTFields dosing}

TTFields dose is defined as the product of TTFields intensity squared, tissuespecific conductivities, and patient usage [52•]. A simulation-based analysis including 340 patients in the EF-14 trial reported significantly longer median PFS ( 8.5 vs 6.7 months; HR 0.70 [95\% CI 0.53-0.92]; $p=0.02$ ) and OS ( 25.2 vs 20.4 months; HR 0.61 [95\% CI $0.46-0.82$ ]; $p=0.003$ ) in patients receiving an average TTFields dose in the tumor bed of $\geq 0.77$ versus $<0.77 \mathrm{~mW} / \mathrm{cm}^{3}$, respectively [52•]. Survival was also significantly longer when the average TTFields intensity in the tumor bed was $\geq 1.06$ versus $<1.06 \mathrm{~V} / \mathrm{cm}$ : median OS was 24.3 versus 21.6 months, respectively (HR 0.69 [95\% CI $0.51-0.94] ; p=0.03)$, and median PFS was 8.1 vs 7.9 months, respectively (HR 0.71 [95\% CI $0.53-0.95] ; p=0.03$ ) [52•]. Increased doses were also associated with improved QoL through longer deteriorationfree survival ( 18.0 vs 9.1 months; HR 0.68 [95\% CI $0.48-0.96] ; p=$ 0.004 ) for doses of $\geq 0.77$ vs $<0.77 \mathrm{~mW} / \mathrm{cm}^{3}$, respectively [52•]. Taken together, this retrospective analysis of the EF-14 study suggests that a higher TTFields dose correlates with improved survival and that simulation models may help optimize TTFields dosimetry and treatment planning.

\section{Clinical management}

\section{Real-world data}

The Patient Registry Dataset (PRiDe) comprised of real-world surveillance data from 457 patients with rGBM treated with TTFields in 91 US centers between October 2011 and November 2013 [63] revealed greater median OS compared with the EF-11 trial (9.6 vs 6.6 months, respectively; HR 0.66 [95\% CI 0.05-0.86]; $p=0.0003$ ] [63]. Notably, survival rates at 1 and 2 years were more than double for TTFields treatment in PRiDe compared with the EF-11 trial (1 year: $44 \%$ vs 20\%; 2 year: $30 \%$ vs $9 \%$, respectively) [63]. Safety and tolerability observed in PRiDe were similar to that demonstrated in the phase EF-11 trial, with $24.3 \%$ of patients in PRiDe reporting array-associated skin reactions and limited systemic AEs [63]. Safety data from a more recent US post-marketing surveillance dataset of 6494 patients with GBM treated with TTFields 
also reported no new safety concerns [69]. The most common AEs were skin reactions (28\%), and general AEs included electric sensation (i.e., tingling sensation) and heat sensation (i.e., local warm feeling beneath the arrays) in $9 \%$ of patients each, pain (any location) in 5\%, and fatigue in $4 \%$ [69]. Skin reactions could be managed with topical treatments and slight shifting of the arrays [69].

\section{Patient management}

After initiating TTFields, treating physicians should monitor monthly usage, to ensure patients receive the maximal treatment benefit and periodically assess the scalp to manage local skin irritation [12]. Standard practice is to perform a follow-up MRI after 2-3 months on treatment [12]. There are unique challenges associated with the ongoing evaluation and interpretation of imaging changes in response to TTFields, which require a longer treatment period ( $\geq 4$ weeks continuous exposure) to reach a state of tumor stabilization [12]. Tumor progression can be challenging to distinguish from pseudoprogression or radiation necrosis, and pseudoresponse should be carefully evaluated in patients receiving combined anti-angiogenic therapy [12]. Retrospective analyses have demonstrated that tumor regression and clinical benefit with TTFields can still occur following early initial radiographic progression $[12,70]$. For example, in an analysis of responders from the EF-11 trial, 43\% experienced progressive disease by 2-24 months following TTFields initiation [70]. However, they subsequently developed delayed (median time to onset of objective response of 8 months) as well as durable (a median duration of 7 months), and objective radiographic responses [12]. Patients with radiographic changes consistent with progression at the 2-month scan, yet who are adherent with therapy and remain neurologically stable, may be maintained on the same array layout with a brain MRI scan repeated after another 2-3 months [12]. For patients at the first follow-up visit with a significant increase of $>25 \%$ in tumor size or in whom new brain lesions appear, it is recommended to replan array layout and treatment [12]. In general, any major changes in imaging from baseline should be reviewed in the context of the TTFields treatment field, and replanning should be considered at the treating physician's discretion.

Within our institution at the University of Florida, TTFields treatment is encouraged for patients outside of clinical trials who fall into 2 categories. The first are patients using TTFields combined with adjuvant maintenance TMZ or salvage therapies such as cytotoxic chemotherapy, compassionate-use immunotherapy, anti-angiogenic therapy, or targeted small molecule therapy. Combinatorial approaches are based on the premise that TTFields offer possible synergistic effects, for example, enhancing BBB and tumor cell permeability [45, 47, 48]. The second category is TTFields used as monotherapy due to patient factors that may limit systemic therapy tolerance such as myelosuppression, decline in functional status, or patient preference.

The authors would like to stress the importance of continuing TTFields when there is suggestion of possible progression within the first several months of 


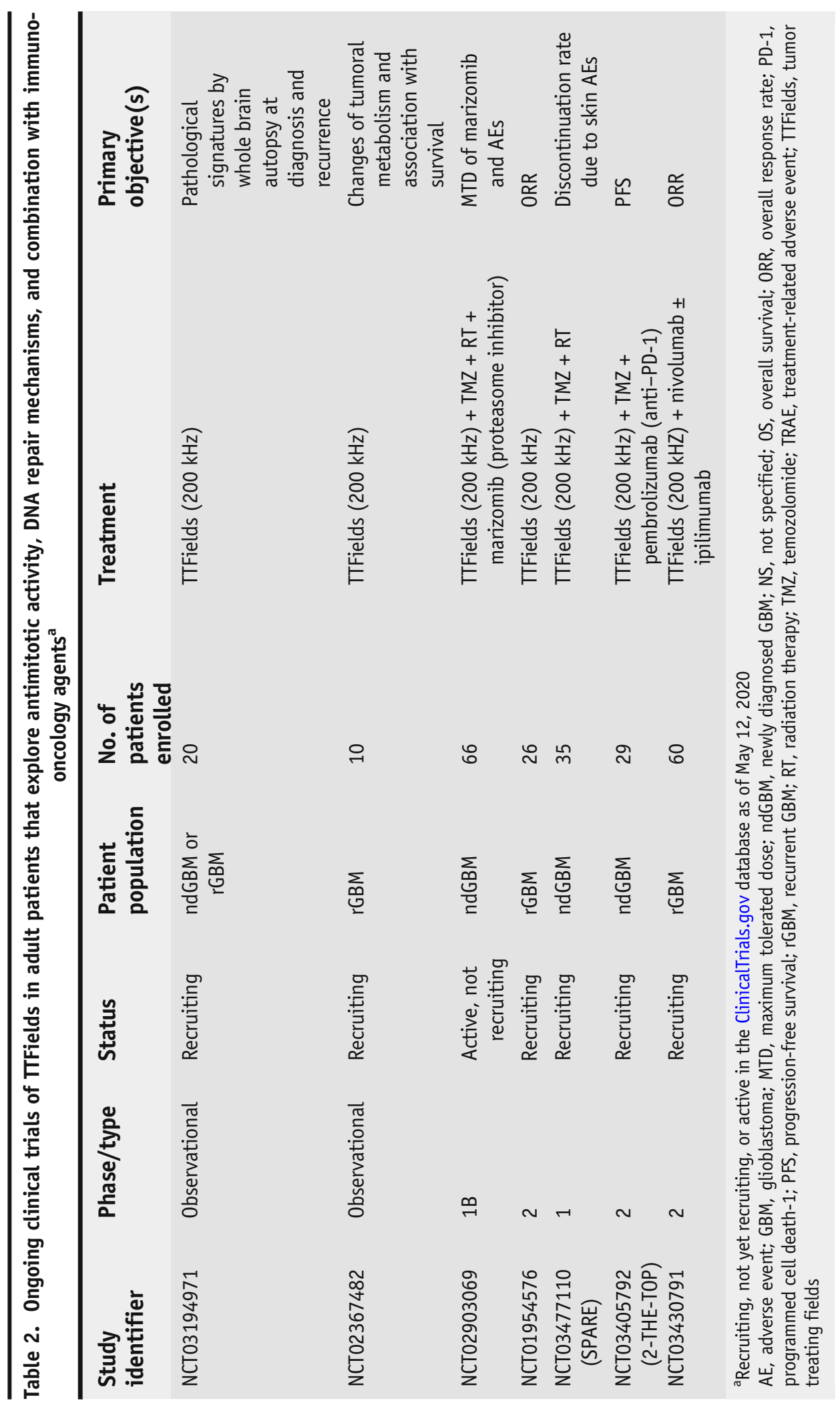


initiation, given the possibility of continued clinical benefit $[12,70]$. This strategy follows the principle of pseudoprogression that can be seen following completion of chemoradiation [12].

\section{Ongoing clinical studies investigating TTFIELDS in glioma}

There is mounting interest in expanding the clinical use of TTFields. Current studies are exploring the role of TTFields in antimitotic activity and DNA repair mechanisms (including DNA methylation status) and combining TTFields with immuno-oncology agents (e.g., pembrolizumab and nivolumab \pm ipilimumab) (Table 2). There is increasing evidence that TTFields can activate the robust innate immunity pathways, including the STING pathway and immunogenic pyroptotic cell death that effectively prime a response by antiprogrammed cell death-1 (PD-1) therapy [40-42]. At our own institution, we are currently evaluating the combination of TTFields, adjuvant TMZ, and pembrolizumab in adult patients with ndGBM in a phase 2, multicenter, single-arm, open-label study (NCT03405792). Preliminary data from our patient cohort compared with the historical controls of TTFields plus adjuvant TMZ and TMZ alone in the EF-14 study have demonstrated a striking survival benefit for the first 7 of 12 currently enrolled patients undergoing this treatment paradigm with a minimum of 12-month follow-up to a median PFS of approximately 12 months [71].

Other studies are exploring clinical efficacy in combination with other agents for GBM and non-GBM gliomas and in broader patient populations (e.g., pediatric, bevacizumab-naive, or bevacizumab-refractory). Studies in low-grade glioma are combining TTFields with bevacizumab; and in GBM, combining TTFields with targeted therapies (e.g., bevacizumab, carvedilol, and marizomib), chemotherapy (e.g., lomustine), cancer vaccines (e.g., personalized mutation-derived tumor antigen vaccine), or personalized surgery (e.g., minor craniotomy or distribution of burr holes). Preliminary data from the Pediatric Brain Tumor Consortium (PBTC)-048 study suggest safety and feasibility of TTFields in pediatric patients with supratentorial high-grade recurrent glioma and ependymoma [72]. Based on preclinical studies demonstrating a role for TTFields in sensitizing tumor cells to RT $[35,36]$, studies evaluating the benefit of TTFields combined with RT in ndGBM are planned. Additionally, studies exploring TTFields response predictors, such as genetic signatures [73] and tumor changes by image analyses, are underway and will provide essential data for stratifying patients likely to respond optimally. Lastly, post-approval studies, like the ongoing multicenter, prospective study of adult patients with ndGBM being conducted in Germany [74], should provide further efficacy and safety data of TTFields treatment in real-world settings.

\section{Neuro-Oncologic Care During the Novel Coronavirus Pandemic and the Role of TIFields}

Given the ongoing novel coronavirus (COVID-19) pandemic, healthcare centers across the globe have had to adjust. We have witnessed temporary shutdowns of elective procedures and conversion of many 
clinical visits to telemedicine. The field of neuro-oncology has not been immune, as patients have been hesitant to travel due to potential exposure concerns to the novel coronavirus. Shutdowns to the research infrastructure of major academic centers have limited the availability of clinical trials due to staff shortages and reduced research capacity. Patients and providers have tried to limit hospital visits as safety precautions. The shifts in treatment delivery have led more providers to consider hypofractionated radiation treatment regimens which commonly utilize 15 fractions of treatment over 3 weeks (providing a total dose of approximately 40-50 Gy) as opposed to the more common schedule of hyperfractionated treatment with 30 fractions of 2 Gy each. Reducing the concurrent schedule ultimately allows for a faster transition of patients to maintenance chemotherapy, which can be managed as outpatient. One consequence of hypofractionated radiation treatment regimens is the possibility that patients may be precluded from certain GBM clinical trials that require the hyperfractionated regimen. The shift in care during the COVID-19 pandemic is an opportunity to utilize

Table 3. Ongoing clinical trials of TrFields treatment in patients with non-glioma tumor types ${ }^{\mathrm{a}}$

\begin{tabular}{|c|c|c|c|c|c|c|}
\hline Identifier & Phase & $\begin{array}{l}\text { Study } \\
\text { name }\end{array}$ & $\begin{array}{l}\text { Patient } \\
\text { population }\end{array}$ & Accrual & Treatment & $\begin{array}{l}\text { Comparator } \\
\text { treatment }\end{array}$ \\
\hline NCT02831959 & 3 & METIS, EF-25 & Brain metastases - NSCLC & 270 & TTFields (150 kHz) after SRS & SoC therapy \\
\hline NCT02973789 & 3 & LUNAR, EF-24 & $\begin{array}{l}\text { Progressive NSCLC after } \\
\text { platinum-based } \\
\text { therapy }\end{array}$ & 534 & $\begin{array}{l}\text { TTFields }(150 \mathrm{kHz})+ \\
\text { docetaxel or immune } \\
\text { checkpoint inhibitors }\end{array}$ & $\begin{array}{l}\text { Docetaxel or immune } \\
\text { checkpoint } \\
\text { inhibitors }\end{array}$ \\
\hline NCT03940196 & 3 & INNOVATE-3, EF-28 & $\begin{array}{l}\text { Recurrent, platinum- } \\
\text { resistant ovarian } \\
\text { carcinoma }\end{array}$ & 540 & $\begin{array}{l}\text { TTFields }(200 \mathrm{kHz})+ \\
\text { paclitaxel }\end{array}$ & Paclitaxel \\
\hline NCT03377491 & 3 & PANOVA-3, EF-27 & $\begin{array}{l}\text { Locally advanced pancreatic } \\
\text { adenocarcinoma }\end{array}$ & 556 & $\begin{array}{l}\text { TTFields }(150 \mathrm{kHz})+ \\
\text { gemcitabine }+ \\
\text { nab-paclitaxel }\end{array}$ & $\begin{array}{l}\text { Gemcitabine + nab- } \\
\text { paclitaxel alone }\end{array}$ \\
\hline NCT03903640 & 2 & NS & $\begin{array}{l}\text { Brain metastases - } \\
\text { melanoma }\end{array}$ & 23 & $\begin{array}{l}\text { TTFields }(200 \mathrm{kHz})+ \\
\text { nivolumab + ipilimumab }\end{array}$ & NA \\
\hline NCT03606590 & 2 & HEPANOVA, EF-30 & $\begin{array}{l}\text { Advanced hepatocellular } \\
\text { carcinoma }\end{array}$ & 25 & $\begin{array}{l}\text { TTFields }(150 \mathrm{kHz})+ \\
\quad \text { sorafenib }\end{array}$ & $\begin{array}{l}\text { Historical sorafenib } \\
\text { alone }\end{array}$ \\
\hline NCT03203525 & 1 & NS & Hepatic metastatic cancer & 52 & $\begin{array}{l}\text { TTFields }(150 \mathrm{kHz})+ \\
\text { FOLFOX } 6 \text { bevacizumab } \\
\text { or liposomal } \\
\text { doxorubicin, } \\
\text { bevacizumab } \\
\text { + temsirolimus }\end{array}$ & NA \\
\hline NCT03488472 & Pilot & NS & Brain metastases - SCLC & 20 & $\begin{array}{l}\text { TTFields (200 kHz) } \\
\text { following SRS }\end{array}$ & NA \\
\hline NCT03995667 & Pilot & NS & Brain metastases - SCLC & 106 & $\begin{array}{l}\text { Prophylactic TTFields } \\
(200 \mathrm{kHz})\end{array}$ & NA \\
\hline NCT03607682 & 2 & NS & Brain metastases - SCLC & 25 & $\begin{array}{l}\text { Prophylactic TTFields } \\
\quad(200 \mathrm{kHz})\end{array}$ & NA \\
\hline
\end{tabular}


TTFields by taking advantage of a modality that is built on care delivered outside of the physician office. In fact, this may be in keeping with the original design of the Phase 3 EF-14 study requiring a total RT dose of 45-70 Gy [26], which encompasses most commonly used hypofractionated regimens. At the time of progressive disease, limited options may make continuation of tumor treating fields a worthwhile option through first recurrence, which also reflects the intent of the EF14 study. Since the outbreak of the novel coronavirus pandemic, remote technical support to patients in uploading the usage report from patient devices and maintaining in-home treatment has allowed TTFields to be a viable treatment option as neuro-oncologists explore providing remote care to their patients.

TTFields have emerged as an important novel treatment modality in GBM management. The clinical efficacy and tolerability of TTFields in GBM were established in 2 large phase 3 trials and supported by realworld data. TTFields are associated with prolonged survival outcomes and minimal AEs, local or systemic, typically associated with other treatment modalities such as RT and chemotherapy, respectively [27, 63]. The most common TTFields-associated AEs are mild-to-moderate array-associated skin AEs [27, 63]. These can typically be managed by shifting the arrays $1-2 \mathrm{~cm}$ and with topical corticosteroids, which do not require substantial treatment breaks and resolve after pause in treatment [75]. While patients may be inconvenienced by regularly shaving their head and adhering to the recommended $75 \%$ time of treatment usage, survival benefits appear to positively correlate with usage level [64]. The ability of TTFields to target and disrupt rapid cancer cell division at multiple phases of the cell cycle allows for combinations with other therapies for synergistic or additive effects [75]. Cell cycle inhibitors and agents targeting cell proliferation, migration, and DNA replication in combination with TTFields are under investigation [76, 77].

The GBM tumor microenvironment has been characterized as being highly immunosuppressive [78]. Treatments currently being explored to reverse intrinsic immunosuppression of GBM include PD-1 inhibitors [79-81], autolytic vaccines [82, 83], dendritic cell vaccines [83], and oncolytic viruses [84-86]. Other GBM treatments under investigation include different RT methods (e.g., photon intensity-modulated, proton beam, and low-dose whole-brain) in place of standard-dose RT and in combination with various targeted agents (e.g., hypoxia-related agents $[76,87]$, epidermal growth factor receptor inhibitors [76, 88-90], proteasome inhibitors [91], and neurotrophin receptor kinase inhibitors [92]). The potential for TTFields to permeabilize the BBB opens the door for combinations with systemic and targeted agents previously 
deemed unfeasible as anti-glioma modalities due to restricted brain access.

Currently, TTFields treatment for supratentorial ndGBM and rGBM is covered by several healthcare insurers [93-95]. Cost is comparable to most novel cancer therapies [96] and was considered to be cost-effective for ndGBM as adjuvant therapy with TMZ compared with TMZ alone, from the US healthcare system perspective, with an incremental costeffectiveness ratio (ICER) of $\sim \$ 150,000$ per life year gained (LYG) [97]. However, global healthcare systems vary, and TTFields were not considered cost-effective from the French healthcare system perspective, with an ICER of $\sim 520,000 €$ per LYG $[98,99]$. In addition to cost, suboptimal knowledge and awareness of TTFields MoA may be reasons for physician hesitation to prescribe TTFields [100]. Although data are accumulating to clarify the MoA of TTFields, ongoing research should help elucidate their impact across different cancer types. In fact, positive, early-phase, clinical trial data suggest potential survival benefits in other tumor types, and clinical trials investigating the efficacy and safety of TTFields in combination with other therapies are underway [101-103]. These include trials evaluating combinations with standard of care therapy in lung, gastrointestinal, ovarian, pancreatic, and hepatocellular cancers, as well as brain metastases (Table 3). Additionally, simulation models of TTFields delivery in spinal regions are being investigated to assess efficacy in non-glioma CNS malignancies [104].

\section{Conclusions}

GBM and malignant glioma are the most common and lethal primary brain cancer in adults. Treatment options for GBM are limited. Interest for TTFields therapy stems from its tolerable safety profile and longer OS compared with the aforementioned Stupp protocol in GBM. Treatment strategies in combination with TTFields provide important clinical benefit while limiting additional toxicity in patients with brain cancer and may extend to patients with other solid tumors.

\section{Acknowledgments}

We would like to acknowledge the following people for their invaluable assistance in this manuscript: Huda Abdullah, PhD, Novocure Ltd., and Katie Groschwitz, PhD, Spark Medica Inc.

\section{Authors' contributions}

All authors contributed to the development of this review; wrote, edited, and reviewed the drafts; and approved the final manuscript. 


\section{Funding information}

This review was funded by the University of Florida, Department of Neurosurgery.

\section{Compliance with Ethical Standards}

\section{Conflicts of Interest}

Ashley P. Ghiaseddin has received research funding from Orbus Therapeutics and has received compensation from Novocure and Monteris Medical for participation on medical advisory boards.

David Shin declares that he has no conflict of interest.

Kaitlyn Melnick declares that she has no conflict of interest.

David D. Tran has received research funding from Novocure, Lacerta Therapeutics, Merck, Novartis, Monteris Medical, Tocagen, Advanced BioScience Laboratories (ABL), and Stemline Therapeutics; has received compensation from Novocure and Monteris Medical for participation on medical advisory boards; has received reimbursement for travel expenses from Novartis; and has the following patents pending:

* "Inhibiting Prostaglandin E Receptor 3 Resensitizes Resistant Cells to TTFields"

* "Methods for Reducing Viability of Cancer Cells by Activation of the STING Pathway with TTFields"

* "AAV capsid variants targeting human glioblastoma stemlike cells" (licensed to Lacerta Therapeutics)

* "Core Master Regulators of Glioblastoma Stem Cells"

* "Methods for Targeted Treatment and Prediction of Patient Survival in Cancer"

* "Methods for Cancer Screening and Monitoring by Cancer Master Regulators Markers in Liquid Biopsy"

* "Immunotherapy for Direct Reprogramming of Cancer Cells into Immune Cells/Antigen Presenting Cells/ Dendritic Cells"

" "GeneRep and nSCORE: Method and Apparatus for Improved Determination of Node Influence in a Network"

\section{References and Recommended Reading}

Papers of particular interest, published recently, have been highlighted as:

- Of importance

- Of major importance

1. Bray F, et al. Global cancer statistics 2018: GLOBOCAN estimates of incidence and mortality worldwide for 36 cancers in 185 countries. CA Cancer J Clin. 2018;68(6):394-424.

2. Patel AP, et al. Global, regional, and national burden of brain and other CNS cancer, 19902016: a systematic analysis for the Global Burden of Disease Study 2016. Lancet Neurol. 2019;18(4):376-93.

3. Ostrom QT, et al. CBTRUS statistical report: Primary brain and other central nervous system tumors diagnosed in the United States in 20122016. Neuro-Oncology. 2019;21(Supplement_5):v1-v100.

4. Mesti T, Ocvirk J. Malignant gliomas: old and new systemic treatment approaches. Radiother Oncol. 2016;50(2):129-38.

5. Garcia CR, et al. Primary central nervous system tumor treatment and survival in the United States, 20042015. J Neuro-Oncol. 2019;144(1):179-91.
6. Velásquez C, et al. Molecular and clinical insights into the invasive capacity of glioblastoma cells. J Oncol. 2019;2019:16.

7. Rosen J, et al. Extracranial metastases of a cerebral glioblastoma: a case report and review of the literature. Case Rep Oncol. 2018;11(2):591-600.

8. Omuro A, DeAngelis LM. Glioblastoma and other malignant gliomas: a clinical review. JAMA. 2013;310(17):1842-50.

9. Kirson ED, et al. Disruption of cancer cell replication by alternating electric fields. Cancer Res. 2004;64(9):3288-95.

10. Kirson ED, et al. Alternating electric fields arrest cell proliferation in animal tumor models and human brain tumors. Proc Natl Acad Sci U S A. 2007;104(24):10152-10,157.

11. Novocure. Optune ${ }^{\circledR}$ : instructions for use. 2019 [cited 2019 July 10]; Available from: https://www.optune. com/Content/pdfs/Optune_IFU_8.5x11.pdf. Accessed 12 May 2020. 
12. Trusheim J, et al. A state-of-the-art review and guidelines for tumor treating fields treatment planning and patient follow-up in glioblastoma. CNS oncology. 2017;6(1):29-43.

13. Stupp R, et al. Radiotherapy plus concomitant and adjuvant temozolomide for glioblastoma. $\mathrm{N}$ Engl J Med. 2005;352(10):987-96.

14. Philip J, et al. A proposed framework of supportive and palliative care for people with high-grade glioma. Neuro-oncology. 2018;20(3):391-9.

15. The National Comprehensive Cancer Network ${ }^{\circledR}$. NCCN clinical practice guidelines in oncology (NCCN Guidelines $^{\circledR}$ ): central nervous system cancers. 2019 10/ 2019 [cited 2019 October 18]; version 2.2020. April 30, 2020 [cited May 12, 2020]: [Available from: https://www.nccn.org/professionals/physician_gls/ pdf/cns.pdf. Accessed 12 May 2020.

16. Kong D-S, et al. Efficacy of stereotactic radiosurgery as a salvage treatment for recurrent malignant gliomas. Cancer. 2008;112(9):2046-51.

17. Cabrera AR, et al. Concurrent stereotactic radiosurgery and bevacizumab in recurrent malignant gliomas: a prospective trial. Int J Radiat Oncol Biol Phys. 2013;86(5):873-9.

18. Wick W, et al. Lomustine and bevacizumab in progressive glioblastoma. N Engl J Med. 2017;377(20):1954-63.

19. Friedman HS, et al. Bevacizumab alone and in combination with irinotecan in recurrent glioblastoma. J Clin Oncol. 2009;27(28):4733-40.

20. Norden AD, et al. Phase 2 study of dose-intense temozolomide in recurrent glioblastoma. Neuro-Oncol. 2013;15(7):930-5.

21. Perry JR, et al. Phase II trial of continuous doseintense temozolomide in recurrent malignant glioma: RESCUE study. J Clin Oncol.

2010;28(12):2051-7.

22. Napoleoni L, et al. Looking for a place for dose-dense TMZ regimens in GBM patients: an experience with MGMT exploratory evaluation. Bioengineering. 2019;6(1):11.

23. Bae $\mathrm{SH}$, et al. Toxicity profile of temozolomide in the treatment of 300 malignant glioma patients in Korea. J Korean Med Sci. 2014;29(7):980-4.

24. Brandes AA, et al. Practical management of bevacizumab-related toxicities in glioblastoma. Oncologist. 2015;20(2):166-75.

25. Simonetti G, et al. Safety of bevacizumab in patients with malignant gliomas: a systematic review. Neurol Sci. 2014;35(1):83-9.

26. Stupp R, et al. Effect of tumor-treating fields plus maintenance temozolomide vs maintenance temozolomide alone on survival in patients with glioblastoma: a randomized clinical trial. JAMA. 2017;318(23):2306-16.

27.• Stupp R, et al. NovoTTF-100A versus physician's choice chemotherapy in recurrent glioblastoma: a randomised phase III trial of a novel treatment modality. Eur J Cancer. 2012;48(14):2192-202
TTFields were shown to be a tolerable targeted treatment modality that when combined with Temozolomide demonstrated efficacy relative to the prior SOC of Temozolomide without increase in systemic AEs.

28. Food and Drug Administration (FDA). Optune $e^{\mathrm{TM}}$ (formerly NovoTTF-100A ${ }^{\mathrm{TM}}$ System) summary of safety and effectiveness data (SSED). 2015 [cited 2020 May 12]; Available from: https://www.accessdata.fda.gov/cdrh_ docs/pdf10/P100034S013b.pdf. Accessed 12 May 2020.

29. Dizon DS, et al. Clinical cancer advances 2016: annual report on progress against cancer from the American Society of Clinical Oncology. J Clin Oncol. 2016;34(9):987-1011.

30. Weinberg U Novocure's tumor treating fields: Innovative brain cancer therapy with survival and safety benefits: introduction to brain cancer research at Novocure. Nature Res, 2018.

31. Karanam NK, et al. Tumor treating fields cause replication stress and interfere with DNA replication fork maintenance: Implications for cancer therapy. Transl Res. 2020;217:33-46.

32. Gera N, et al. Tumor treating fields perturb the localization of septins and cause aberrant mitotic exit. PLoS One. 2015;10(5):e0125269.

33. Mun EJ, et al. Tumor-treating fields: a fourth modality in cancer treatment. Clin Cancer Res. 2018;24(2):26675.

34. Wenger $\mathrm{C}$, et al. The electric field distribution in the brain during TTFields therapy and its dependence on tissue dielectric properties and anatomy: a computational study. Phys Med Biol. 2015;60(18):7339-57.

35. Giladi M, et al. Tumor treating fields (TTFields) delay DNA damage repair following radiation treatment of glioma cells. Radiat Oncol. 2017;12(1):206.

36. Karanam NK, et al. Tumor-treating fields elicit a conditional vulnerability to ionizing radiation via the downregulation of BRCA1 signaling and reduced DNA double-strand break repair capacity in non-small cell lung cancer cell lines. Cell Death Dis. 2017;8(3):e2711.

37. Karanam NK, et al., Tumor treating fields cause replication stress and interfere with DNA replication fork maintenance: implications for cancer therapy. Transl Res, 2019

38. Shteingauz A, et al. AMPK-dependent autophagy upregulation serves as a survival mechanism in response to Tumor Treating Fields (TTFields). Cell Death Dis. 2018;9(11):1074.

39. Wong ET, Timmons J, Swanson KD. Abstract 1707: Tumor treating fields exert cellular and immunologic effects. Cancer Res. 2018;78(13 Supplement):1707.

40. Giladi M, et al. Alternating electric fields (TTFields) induce immunogenic cell death resulting in enhanced antitumor efficacy when combined with anti-PD-1 therapy. J Immunol. 2016;196(1 Supplement):75.26.

41. Kaynan, N., et al., P189: Tumor Treating Fields (TTFields) induce immunogenic cell death resulting in enhanced antitumor efficacy when combined with anti-PD-1 therapy. J Immunother Cancer, 2019. 
TTFields activating the STING pathway has provided checkpoint immunotherapy drugs an attractive modality to enhance effectiveness. Combination approaches for immune checkpoint drugs are needed given the lack of reported benefit using anti-PD1 therapy as monotherapy in GBM.

42. Chen D, et al. IMMU-06. TTFields induces immunogenic cell death and STING pathway activation through cytoplasmic double-stranded DNA in glioblastoma cells. Neuro-Oncology.

2019;21(Supplement_6):vi120.

43. Kirson ED, et al. Alternating electric fields (TTFields) inhibit metastatic spread of solid tumors to the lungs. Clin Exp Metastasis. 2009;26(7):633-40.

44. Schneiderman RS, et al. ANGI-11. Tumor treating fields (TTFields) inhibit cancer cell migration and invasion by inducing reorganization of the actin cytoskeleton and formation of cell adhesions. Neuro-Oncology. 2018;20(suppl_6):vi30.

45. Kim EH, et al. Tumor treating fields inhibit glioblastoma cell migration, invasion and angiogenesis. Oncotarget. 2016;7(40):65125-65,136.

46. Silginer $\mathrm{M}$, et al. Biological activity of tumor-treating fields in preclinical glioma models. Cell Death Dis. 2017;8(4):e2753.

47. Kessler AF, et al. Abstract 252: Tumor treating fields (TTFields) affect blood brain barrier (BBB) integrity in vitro and in vivo. Cancer Res. 2019;79(13 Supplement):252.

48.• Chang E, et al. Tumor treating fields increases membrane permeability in glioblastoma cells. Cell Death Dis. $2018 ; 4(1): 113$

TTFields demonstrating transient and reversible increase in membrane permeability of GBM cells highlights the benefit of adding this modaligy to SOC, salvage, and novel treatment options.

49. Winkler F, Osswald M, Wick W. Anti-angiogenics: their role in the treatment of glioblastoma. Oncol Res Treat. 2018;41(4):181-6.

50. Kochar AS, et al. Contemporary updates on clinical trials of antiangiogenic agents in the treatment of glioblastoma multiforme. Asian J Neurosurg. 2018;13(3):546-54.

51. Porat $\mathrm{Y}$, et al. Determining the optimal inhibitory frequency for cancerous cells using tumor treating fields (TTFields). J Vis Exp. 2017;123.

52. Ballo MT, et al. Correlation of tumor treating fields dosimetry to survival outcomes in newly diagnosed glioblastoma: a large-scale numerical simulation-based analysis of data from the phase $3 \mathrm{EF}-14$ randomized trial. Int J Radiat Oncol Biol Phys. 2019;104(5):110613

Relates TTFields usage to survival benefit, emphasizing the importance of achieving high usage during treatment.

53. Korshoej AR, et al. Impact of tumor position, conductivity distribution and tissue homogeneity on the distribution of tumor treating fields in a human brain: a computer modeling study. PLoS One.

2017;12(6):e0179214.
54. Bomzon $\mathrm{Z}$, et al. Using computational phantoms to improve delivery of tumor treating fields (TTFields) to patients. Conf Proc IEEE Eng Med Biol Soc.

2016;2016:6461-4.

55. Wenger $\mathrm{C}$, et al. Improving tumor treating fields treatment efficacy in patients with glioblastoma using personalized array layouts. Int J Radiat Oncol Biol Phys. 2016;94(5):1137-43.

56. Miranda PC, et al. Predicting the electric field distribution in the brain for the treatment of glioblastoma. Phys Med Biol. 2014;59(15):4137-47.

57. Wenger C, et al. NIMG-41. Rapid and accurate creation of patient-specific computational models for GBM patients receiving Optune therapy with conventional imaging (T1w/PD). Neuro-Oncology.

2019;21(Supplement_6):vi170.

58. Naveh A, Yesharim O, Bomzon ZE. RDNA-10. TTFields treatment planning for targeting multiple lesions spread throughout the brain. Neuro-Oncology. 2019;21(Supplement_6):vi208-9.

59. Bomzon $\mathrm{Z}$, et al. Modelling tumor treating fields for the treatment of lung-based tumors. Conf Proc IEEE Eng Med Biol Soc. 2015;2015:6888-91.

60. Connelly J, et al. Planning TTFields treatment using the NovoTAL system-clinical case series beyond the use of MRI contrast enhancement. BMC Cancer. 2016;16(1):842.

61. Chaudhry A, et al. NovoTTF-100A system (tumor treating fields) transducer array layout planning for glioblastoma: a NovoTAL system user study. World J Surg Oncol. 2015;13:316.

62. Taphoorn MJB, et al. Influence of treatment with tumor-treating fields on health-related quality of life of patients with newly diagnosed glioblastoma: a secondary analysis of a randomized clinical trial. JAMA Oncol. 2018;4(4):495-504.

63. Mrugala MM, et al. Clinical practice experience with NovoTTF-100A ${ }^{\mathrm{TM}}$ system for glioblastoma: the patient registry dataset (PRiDe). Semin Oncol. 2014;41:S4S13.

64. Kanner AA, et al. Post hoc analyses of intention-to-treat population in phase III comparison of NovoTTF$100 \mathrm{~A}^{\mathrm{TM}}$ system versus best physician's choice chemotherapy. Semin Oncol. 2014;41:S25-34.

65. Benson L. Tumor Treating Fields technology: alternating electric field therapy for the treatment of solid tumors. Semin Oncol Nurs. 2018;34(2):137-50.

66. Toms SA, et al. Increased compliance with tumor treating fields therapy is prognostic for improved survival in the treatment of glioblastoma: a subgroup analysis of the EF-14 phase III trial. J Neuro-Oncol. 2019;141(2):467-73.

67. Novocure. Explore Optune ${ }^{\circledR} .2019$ [cited 2020 May 12]; Available from: https://www.optune.com/ discover-optune/explore-the-system. Accessed 12 May 2020.

68. Kinzel A, et al. Tumor treating fields for glioblastoma treatment: patient satisfaction and compliance with the 
second-generation Optune((R)) system. Clin Med Insights Oncol. 2019;13:1-7.

69. Tran D. NCMP-21. Real-world surveillance data for tumor treating fields affirm the tolerability of tumor treating fields for the treatment of glioblastoma in the United States. Neuro-Oncology.

2018;20(suppl_6):vi198.

70. Wong ET, et al. Response assessment of NovoTTF-100A versus best physician's choice chemotherapy in recurrent glioblastoma. Cancer Med. 2014;3(3):592-602.

71. Tran D, et al. ATIM-39. Phase 2 open-labeled study of adjuvant temozolomide plus tumor treating fields plus pembrolizumab in patients with newly diagnosed glioblastoma (2-THE-TOP). Neuro-Oncology. 2019;21(Supplement_6):vi10.

72. Goldman S, et al. PDCT-07. Feasibility trial of TTFields (tumor treating fields) for children with recurrent or progressive supratentorial high-grade glioma (HGG) and ependymoma: a pediatric brain tumor consortium study: PBTC-048. Neuro-Oncology.

2018;20(suppl_6):vi201.

73. Tran D, et al. PATH-27. Identifying the genetic signature of response in a phase II study of tumor treating fields in recurrent glioblastoma. Neuro-Oncology. 2018;20(suppl_6):vi164.

74. Bähr O, et al. ACTR-31. The use of TTFields for newly diagnosed GBM patients in Germany in routine clinical care (TIGER: TTFields in Germany in routine clinical care). Neuro-Oncology. 2019;21(Supplement_6):vi20.

75. Wong ET, Toms SA, Ahluwalia MS. Technological advances in the treatment of cancer: combining modalities to optimize outcomes. Clin Adv Hematol Oncol. 2015;13(Suppl_11):1-18.

76. NIH: National Cancer Institute. Clinical trials to treat adult glioblastoma. 2019 [cited 2020 May 12]; Available from: https://www.cancer.gov/about-cancer/ treatment/clinical-trials/adult-glioblastoma. Accessed 12 May 2020.

77. Chen Z-P, et al. ACTR-71. Phase $1 / 2$ study of dianhydrogalactitol (VAL-083) with radiation therapy in patients with newly diagnosed, MGMT-unmethylated glioblastoma. Neuro-Oncology.

2018;20(suppl_6):vi27.

78. Nduom EK, Weller M, Heimberger AB. Immunosuppressive mechanisms in glioblastoma. Neuro-oncology. 2015;17(Suppl 7):vii9-vii14.

79. Weinberg U, et al. 187TiP - Tumor Treating Fields concurrent with standard of care therapy for stage 4 NSCLC following platinum failure: phase 3 LUNAR study. Ann Oncol. 2019;30(Supplement_2):ii38-68.

80. Chen RQ, et al. The prognostic and therapeutic value of PD-L1 in glioma. Front Pharmacol. 2019;9:1503.

81. Mantica M, et al. Retrospective study of nivolumab for patients with recurrent high grade gliomas. J NeuroOncol. 2018;139(3):625-31.

82. Imvax I Imvax announces positive results from clinical trial of novel IGV-001 autologous cell vaccine in treating patients with newly diagnosed glioblastoma. 2019 [cited 2020 May 12]; Available from: https://www. globenewswire.com/news-release/2019/03/31/ 1790266/0/en/Imvax-Announces-Positive-Resultsfrom-Clinical-Trial-of-Novel-IGV-001-AutologousCell-Vaccine-in-Treating-Patients-with-NewlyDiagnosed-Glioblastoma.html. Accessed 12 May 2020.

83. Liau LM, et al. First results on survival from a large phase 3 clinical trial of an autologous dendritic cell vaccine in newly diagnosed glioblastoma. J Transl Med. 2018;16(1):142.

84. Desjardins A, et al. Recurrent glioblastoma treated with recombinant poliovirus. $\mathrm{N}$ Engl J Med.

2018;379(2):150-61.

85. Zhu Z, et al. Zika virus has oncolytic activity against glioblastoma stem cells. J Exp Med. 2017;214(10):2843-57.

86. Lang FF, et al. Phase I Study of DNX-2401 (Delta-24RGD) Oncolytic adenovirus: replication and immunotherapeutic effects in recurrent malignant glioma. J Clin Oncol. 2018;36(14):1419-27.

87. Gainer J, Cook H. ACTR-70. Increasing survival in biopsy-only GBM patients. Neuro-Oncol. 2018;20(suppl_6):vi27.

88. Stepanenko AA, Chekhonin VP. Recent advances in oncolytic virotherapy and immunotherapy for glioblastoma: a glimmer of hope in the search for an effective therapy? Cancers. 2018;10(12):492.

89. Gan HK, et al. Safety, pharmacokinetics, and antitumor response of depatuxizumab mafodotin as monotherapy or in combination with temozolomide in patients with glioblastoma. NeuroOncol. 2018;20(6):838-47.

90. Reardon DA, et al. Efficacy and safety results of ABT414 in combination with radiation and temozolomide in newly diagnosed glioblastoma. Neuro-oncology. 2017;19(7):965-75.

91. Roth P, et al. EORTC 1709/CCTG CE.8: A phase III trial of marizomib in combination with standard temozolomide-based radiochemotherapy versus standard temozolomide-based radiochemotherapy alone in patients with newly diagnosed glioblastoma. J Clin Oncol. 2019;37(15_suppl):TPS2072.

92. Ziegler DS, et al. Brief Report: Potent clinical and radiological response to larotrectinib in TRK fusiondriven high-grade glioma. Br J Cancer. 2018;119(6):693-6.

93. Premera ${ }^{\circledR}$. Medical policy - 1.01.29: tumor treating fields therapy. 2019 [cited 2020 May 12]; Available from: https://www.premera.com/medicalpolicies/1. 01.29.pdf. Accessed 12 May 2020.

94. UnitedHealthcare ${ }^{\circledR}$. UnitedHealthcare commercial: medical policy - electric tumor treatment field therapy. 2019 [cited 2020 May 12]; Available from: https:// www.uhcprovider.com/content/dam/provider/docs/ public/policies/comm-medical-drug/electric-tumortreatment-field-therapy.pdf. Accessed 12 May 2020.

95. commonwealth care alliance ${ }^{\circledR}$. Medical necessity guideline (MNG) title: electric tumor treatment field therapy. 
2019. https://www.commonwealthcarealliance.org/ getmedia/7cb11576-ea8b-4719-8a32-17f629b653be/ Electric-Tumor-Treatment-Field-Therapy-MNG-003.

Accessed 12 May 2020.

96. Mehta $M$, et al. Critical review of the addition of tumor treating fields (TTFields) to the existing standard of care for newly diagnosed glioblastoma patients. Crit Rev Oncol Hematol.

$$
\text { 2017;111:60-5. }
$$

97. Guzauskas GF, et al. Tumor treating fields and maintenance temozolomide for newly-diagnosed glioblastoma: a cost-effectiveness study. J Med Econ. 2019;22(10):1006-13.

98. Connock $\mathrm{M}$, et al. Cost-effectiveness of tumor-treating fields added to maintenance temozolomide in patients with glioblastoma: an updated evaluation using a partitioned survival model. J Neuro-Oncol. 2019;143:605-11.

99. Bernard-Arnoux F, et al. The cost-effectiveness of tumor-treating fields therapy in patients with newly diagnosed glioblastoma. Neuro-Oncology. 2016;18(8):1129-36.

100. Fabian D, et al. Treatment of glioblastoma (GBM) with the addition of tumor-treating fields (TTF): a review. Cancers. 2019;11(2):174.
101. Pless $\mathrm{M}$, et al. A phase $\mathrm{I} / \mathrm{II}$ trial of tumor treating fields (TTFields) therapy in combination with pemetrexed for advanced non-small cell lung cancer. Lung Cancer. 2013;81(3):445-50.

102. Rivera F, et al. Tumor treating fields in combination with gemcitabine or gemcitabine plus nab-paclitaxel in pancreatic cancer: results of the PANOVA phase 2 study. Pancreatology. 2019;19(1):64-72.

103. Vergote I, et al. Tumor Treating Fields in combination with paclitaxel in recurrent ovarian carcinoma: results of the INNOVATE pilot study. Gynecol Oncol. 2018;150(3):471-7.

104. Naveh A, Yesharim O, Bomzon ZE. EXTH-37. A novel transducer array layout for delivering tumor treating fields to the spine. Neuro-Oncology.

2019;21(Supplement_6):vi90.

\section{Publisher's note}

Springer Nature remains neutral with regard to jurisdictional claims in published maps and institutional affiliations. 